\title{
Manifestações clínicas orais da sífilis
}

\author{
Oral clinical manifestations of syphilis
}

Bárbara Capitanio de Souza*

\section{Resumo}

Objetivo: discutir as manifestações clínicas orais da sífilis, com a apresentação de casos clínicos. Relato de caso: Caso 1: paciente masculino, 28 anos, apresentando placa mucosa elevada, firme ao toque, de superfície irregular e com cobertura fibrinóide, contornos bem definidos, sem sintomatologia, na mucosa do lábio inferior. Também foi observado um nódulo bilobulado, firme ao toque, superfície irregular e com cobertura fibrinóide, contornos bem definidos, sem sintomatologia, no ápice de língua. Caso 2: paciente masculino, 22 anos, apresentando queixa de dor de garganta, febre baixa $\left(38^{\circ} \mathrm{C}\right)$, mal estar e linfoadenopatia cervical, há 15 dias. O exame clínico oral revelou duas áreas erosivas, eritematosas, sem sintomatologia, no dorso da língua, e uma lesão ulcerada rasa, circundada por área eritematosa, na borda da língua. Foram solicitados exames laboratoriais para os pacientes, confirmando o diagnóstico de sífilis secundária. O tratamento recomendado foi iniciado, havendo a regressão dos sinais clínicos e sintomas, a partir da segunda semana de tratamento. Considerações finais: o desafio do diagnóstico reside no reconhecimento das manifestações clínicas da sífilis, incluindo as características das lesões mucosas e cutâneas. O cirurgião-dentista tem um importante papel de contribuição, devendo conhecer as manifestações mais comuns de sífilis na mucosa oral, para auxiliar com efetividade no diagnóstico e tratamento da doença.

Palavras-chave: infecções por treponema; diagnóstico bucal; sífilis.

\section{Introdução}

A sífilis é uma doença sistêmica, infecciosa, causada pela bactérica anaeróbia Treponema pallidum. A doença pode afetar qualquer órgão do corpo e sem o tratamento adequado pode resultar em problemas neurológicos, cardiovasculares ou ósseos ${ }^{1}$. A sífilis foi uma doença bastante presente na sociedade, mas com a introdução da penicilina e das campanhas de prevenção, a doença sofreu um declínio de sua prevalência e incidência. Nos últimos anos, mesmo havendo protocolos e recomendações bem estabelecidos, houve um ressurgimento significativo da sífilis, em muitos países ${ }^{2}$.

A sífilis apresenta três estágios clínicos. O estágio primário da doença pode se manifestar em aproximadamente 90 dias após a exposição e ter uma remissão espontaneamente dentro de 2 a 8 semanas. A sífilis primária se caracteriza com uma lesão inicial, cancro, que se desenvolve no local de exposição. O estágio secundário ocorre entre 2 e 12 semanas após a exposição inicial. Esta fase é resultante da disseminação hematológica e linfática da infecção $0^{3,4}$. A apresentação usual da doença secundária envolve sintomas cutâneos, mucosos e sistêmicos, dor de cabeça, febre baixa, anorexia, perda de peso e aumento dos linfonodos. Muitas vezes não há uma clara demarcação entre as fases primária e secundária, e um cancro primário pode estar presente em alguns pacientes com sífilis secundária. O estágio terciário ou tardio da sífilis pode se manifestar em 3 anos 
ou mais, após a exposição. Nesta fase pode haver o comprometimento do sistema nervoso central ${ }^{1,2,4}$.

As lesões orais da sífilis podem ser múltiplas e com características diversas, o que aumenta a complexidade do diagnóstico ${ }^{3}$. As manifestações mais comuns são placas cinzentas, úlceras com bordas irregulares e esbranquiçadas, placas mucosas, nódulos, manchas e erosão. A sífilis apresenta aspecto clínico inespecífico sendo mimetizadora de outras condições; portanto, é fundamental que o profissional conheça os possíveis diagnósticos diferenciais. A análise do exame clínico associado com exame físico e ensaios sorológicos, normalmente permite o diagnóstico da doença ${ }^{4}$. O cirurgião-dentista deve conhecer as manifestações mais comuns de sífilis na mucosa oral, para auxiliar no diagnóstico e tratamento da doença. Este trabalho relata dois casos de manifestação oral de sífilis em paciente adulto.

\section{Relato dos Casos}

\section{Caso 1}

Paciente masculino, 28 anos, foi encaminhado para um serviço de estomatologia devido a queixa de aparecimento de lesões orais, com evolução de 45 dias. Durante o exame clínico preliminar, foi observada a presença de uma placa mucosa elevada, firme ao toque, de superfície irregular e com cobertura fibrinóide, contornos bem definidos, sem sintomatologia, na mucosa do lábio inferior, medindo $25 \times 10 \mathrm{~mm}$ (Figura 1A). Também foi observado um nódulo bilobulado, firme ao toque, superfície irregular e com cobertura fibrinóide, contornos bem definidos, sem sintomatologia, no ápice de língua, medindo $20 \times 15 \mathrm{~mm}$ (Figura 1B). O histórico médico não apresentava particularidades. Para diagnóstico diferencial, foi realizado teste rápido para sífilis, o qual foi positivo. Como seguimento protocolar, foram solicitados exames laboratoriais para HIV, hepatites $\mathrm{B}$ e $\mathrm{C}$ e o exame venereal disease research laboratory (VDRL), confirmatório para sífilis. O exame VDRL foi positivo (1:128), confirmando o diagnóstico da doença. Os demais resultados foram negativos.

\section{Caso 2}

Paciente masculino, 22 anos, foi encaminhado para um serviço de estomatologia devido a queixa de aparecimento de lesões orais, com evolução de 30 dias. $\mathrm{O}$ paciente apresentava queixa de dor de garganta, febre baixa $\left(38^{\circ} \mathrm{C}\right)$, mal estar e linfoadenopatia cervical, há 15 dias. O exame clínico oral revelou duas áreas erosivas, eritematosas, sem sintomatologia, no dorso da língua, a maior medindo 7 mm de diâmetro (Figura 1C). Foi observada, também, uma lesão ulcerada rasa, circundada por área eritematosa, na borda da língua, medindo $12 \mathrm{~mm}$ de diâmetro (Figura 1D), com a presença de placa cinzenta, irregular, adjacente à região de ulceração (Figura 1D seta). O paciente relatou que, no último ano, foi diagnosticado com sífilis, mas não realizou o tratamento conforme indicado. Devido às características das lesões e o histórico médico do paciente, foi realizado teste rápido para sífilis, o qual foi positivo. Igualmente ao caso anterior, como seguimento protocolar, foram solicitados exames laboratoriais para HIV, hepatites B e C e o exame VDRL, o qual foi positivo (1:64), confirmando o diagnóstico da doença. Os demais resultados foram negativos.
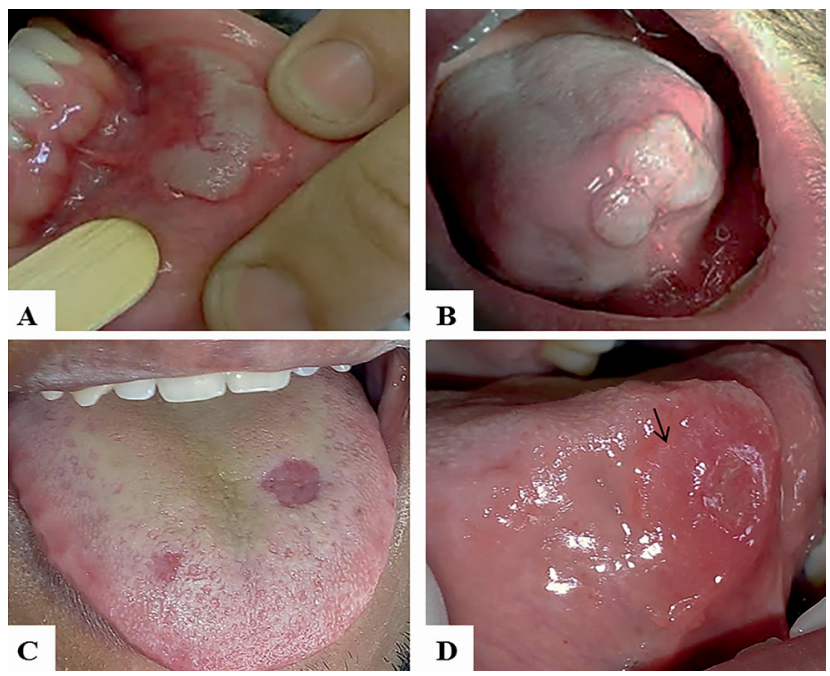

Figura 1 - Aspecto clínico das lesões orais. Placa mucosa elevada firme ao toque, com superfície fibrinóide e irregular (A). Nódulo bilobulado, firme ao toque, superfície irregular e cobertura fibrinóide (B). Áreas erosivas, eritematosas, consistência semelhante á mucosa, contornos bem definidos (C). Lesão ulcerada rasa, recoberta por membrana fibrinolítica, circundada por área eritematosa (D). Placa cinzenta, irregular, consistência semelhante á mucosa, adjacente à região de ulceração ( $D$ seta).

Após a confirmação do diagnóstico de sífilis secundária, os pacientes iniciaram o tratamento com injeções de penicilina $\mathrm{G}$ benzatina, 2,400,000 UI/semana, durante três semanas. Os pacientes também foram instruídos quanto a práticas sexuais seguras, o risco de reinfecção quando os parceiros sexuais não são tratados para a doença, e o risco de adquirir HIV e outras DSTs.

\section{Discussão}

O Treponema pallidum, causador da sífilis, tem o homem como único hospedeiro conhecido e não pode sobreviver fora de seu anfitrião natural, pois tem capacidades metabólicas limitadas, para sintetizar seus próprios bionutrientes ${ }^{5}$. A transmissão da sífilis pode ocorrer de modo vertical (sífilis congênita), quando há contágio in útero ou durante o parto, se o recém-nascido entrar em contato com uma lesão contagiosa ou sexual (sífilis adquirida), embora a transmissão indireta também possa acontecer através de objetos contaminados, como agulhas de tatuagem ou transfusão de sangue ${ }^{5,6}$. $\mathrm{O}$ principal modo de transmissão da sífilis é o contato 
sexual. Após o Treponema pallidum penetrar através da mucosa genital ou pele, ele entra na corrente linfática e sanguínea e se dissemina para os órgãos, incluindo o sistema nervoso central ${ }^{5,7}$. Além disso, pode haver a transmissão da doença por sexo oral, beijo e contato próximo com uma lesão infecciosa ${ }^{6}$.

As manifestações orais são incomuns e podem representar um desafio diagnóstico devido ao seu amplo espectro de aparências clínicas ${ }^{1,8}$. As características da doença pode imitar outras condições e devido sua incidência crescente em muitas partes do mundo, ela deve ser considerada no diagnóstico diferencial de lesões orais ${ }^{3}$. A manifestação oral primária aparece no local da infecção e é normalmente é caracterizada por lesão ulcerada. A lesão pode começar como uma pápula que pode evoluir para uma úlcera endurecida, indolor, não purulenta e de base limpa. O tamanho das lesões é variável e as margens aparecem normalmente delimitadas. Os locais anatômicos mais afetados são principalmente a língua, a gengiva, o palato mole e os lábios. As lesões iniciais podem ser únicas ou múltiplas e geralmente apresentam regressão de 2 a 8 semanas, ainda que sem tratamento, podendo haver uma falsa impressão de resolução da doença ${ }^{3,9}$.

$\mathrm{Na}$ etapa secundária da sífilis pode haver manifestações em áreas de mucosa e pele. As erupções cutâneas se desenvolvem como máculas simétricas rosadas ou vermelhas, que podem evoluir para a forma papular ou pustular ${ }^{10}$. Os pacientes dos casos apresentados não manifestavam lesões cutâneas. Já as manifestações orais da doença são bastante variáveis. Nesta fase é comum o aparecimento de manchas ou placas mucosas ligeiramente elevadas e cobertas por pseudomembranas brancas ou acinzentadas. Estas lesões podem, ainda, estar associadas a áreas eritematosas ${ }^{11}$. Lesões ulceradas, semelhantes às lesões primárias, com bordas irregulares e esbranquiçadas, também podem ser observadas. Podem ocorrer, ainda, máculas vermelhas ou áreas erosivas na mucosa, lesões nodulares e maculopapulares. O condiloma lata é um dos sinais característicos da sífilis secundária, que pode aparecer na mucosa, como no primeiro caso apresentado. Esta lesão é raramente relatada na cavidade oral ${ }^{12}$.

Os pacientes também podem relatar sinais e sintomas semelhantes a uma gripe, envolvendo dor de garganta ou rouquidão devido á inflamação da faringe, da laringe ou das amígdalas. Nestes casos, pode haver linfoadenopatia cervical, como relatado no segundo caso apresentado ${ }^{7}$. O envolvimento do sistema nervoso central, como sintomas cognitivos, ataxia e paralisia, pode ocorrer em todas as fases, mas está mais associado à sífilis terciária. Neste estágio também pode ser observado um quadro de glossite generalizada ${ }^{2}$.

Exames sorológicos em combinação com um exame clínico completo são fundamentais para o diagnóstico da doença ${ }^{7}$. O tratamento empírico prévio ao diagnóstico pode dificultar a identificação de doen- ças infecciosas e promover a disseminação intraindividual dos microorganismos. Se não for diagnosticado no primeiro ou segundo estágio, o paciente pode ficar submetido ao risco de complicações relacionado à sífilis terciária. Estas manifestações são um alerta para um diagnóstico adequado e rápido ${ }^{2}$. Além disso, os períodos extensos de latência, que podem ocorrer, podem dar a falsa impressão de tratamento bem sucedido. Assim, é importante, também, um acompanhamento sorológico para confirmação do sucesso do tratamento ${ }^{13}$. $\mathrm{O}$ dentista também pode desempenhar um papel importante no controle da sífilis, pela identificação dos sinais e sintomas da doença, educação do paciente e encaminhamentos.

\section{Considerações finais}

A sífilis ainda se apresenta como um problema de saúde pública em todo o mundo, mesmo havendo grandes avanços em relação á prevenção e ao tratamento da doença. O desafio do diagnóstico reside no reconhecimento das manifestações clínicas da sífilis, incluindo as características das lesões mucosas e cutâneas. A suspeita de sífilis deve ser considerada na avaliação das lesões orais, especialmente quando há história de contato sexual desprotegido. $\mathrm{O}$ cirurgião-dentista tem um importante papel de contribuição, devendo conhecer as manifestações mais comuns de sífilis na mucosa oral, para auxiliar com efetividade no diagnóstico e tratamento da doença.

\section{Abstract}

Objective: to discuss the oral clinical manifestations of syphilis by presenting clinical cases. Case report: Case 1: male patient, 28 years old, presenting high mucosal plaque that was firm to the touch, with irregular surface and fibrinoid cap, well-defined contours, asymptomatic, and located on the lower lip mucosa. It was also observed a bilobed nodule that was firm to the touch, with irregular surface and fibrinoid cap, well-defined contours, asymptomatic, and located on the tongue. Case 2: male patient, 22 years old, complaining of sore throat, mild fever (38oC), malaise, and cervical lymphadenopathy, 15 days prior. The oral clinical examination showed two erosive and erythematous areas, asymptomatic, and located on the back of the tongue, as well as a shallow ulcerated lesion surrounded by an erythematous area, on the edge of the tongue. Laboratory tests were requested for the patients, confirming the diagnosis of secondary syphilis. The recommended treatment was initiated, and the clinical signs and symptoms receded after the second week of treatment. Final considerations: the diagnosis challenge is recognizing the clinical manifestations of syphilis, including the characteristics of mucosal and cutaneous lesions. Dentists play an important role of contribution and should know the most common manifestations of syphilis in the oral mucosa, in order to effectively assist the diagnosis and treatment of the disease.

Keywords: Treponemal infections. Oral diagnosis. Syphilis. 


\section{Referências}

1. Little JW. Syphilis: an update. Oral Surg Oral Med Oral Pathol Oral Radiol Endodontol 2005;100:3-9.

2. Ficarra G, Roman C. Syphilis: The Renaissance of an Old Disease with Oral Implications. Head Neck Pathol 2009;3(3):195-206.

3. Noronha ACC, Israel MS, Almeida DCF, Moreira GM, Lourenc „o SQC, Dias EP. Sífilis secundária: diagnóstico a partir das lesões orais. DST - J Bras Doenças Sex Transm 2006;18:190-193.

4. Ramoni S, Cusini M, Crosti FGC. Syphilitic chancres of the mouth: three cases. Acta Derm Venereol 2009;89:648-649.

5. Sanchez MR. Syphilis. In: Wolff K, Goldsmith LA, Katz SI, Gilchrest BA, Paller AS, Leffell DJ, editors. Fitzpatrick's dermatology in general medicine 2008;1955-1977.

6. Kent ME, Romanelli F. Reexamining syphilis: an update on epidemiology, clinical manifestations, and management. Ann Pharmacother 2008;42:22-36.

7. Ikenberg K, Springer E, Bräuninger W, Kerl K, Mihic D, Schmid S, Schmitt A, Yeginsoy S, Bode B, Weber A. Oropharyngeal lesions and cervical lymphadenopathy: syphilis is a differential diagnosis that is still relevant. J Clin Pathol 2010;63(8):731-736.

8. Seibt CE, Munerato MC. Secondary syphilis in the oral cavity and the role of the dental surgeon in STD prevention, diagnosis and treatment: a case series study. Braz J Infect Dis 2016;20(4):393-839.

9. Domantay-Apostol GP, Handog EB, Gabriel MTG. Syphilis: the international challenge of the great imitator. Dermatol Clin 2008;26:191-202.

10. Bruce AJ, Rogers RS. Oral manifestations of sexually transmitted diseases. Clin Dermatol 2004;22:520-527.

11. Scott CM, Flint SR. Oral syphilis-re-emergence of an old disease with oral manifestations. Int $\mathrm{J}$ Oral Maxillofac Surg 2005;34:58-63.

12. Ulmer A, Fierlbeck G. Images in clinical medicine. Oral manifestations of secondary syphilis. $N$ Engl $J$ Med 2002;347:1677.

13. Dybeck Udd S, Lund B. Oral Syphilis: A Reemerging Infection Prompting Clinicians' Alertness. Case Rep Dent 2016. Doi: 10.1155/2016/6295920.

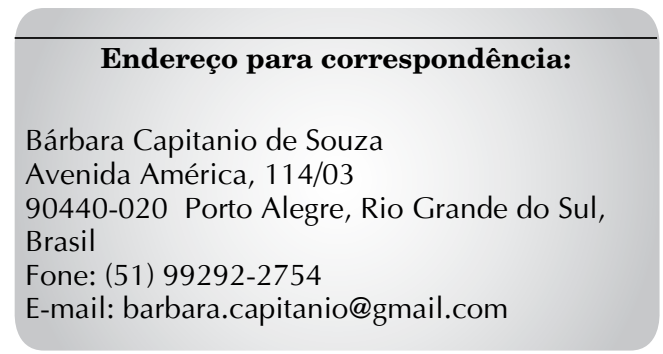

Recebido: 03/12/2016. Aceito: 02/03/2017. 